\title{
Evaluation of a SiPM array detector coupled to a LFS-3 pixellated scintillator for PET/MR applications
}

\author{
Stratos David ${ }^{1 *}$, Eleftherios Fysikopoulos ${ }^{1}$, Maria Georgiou ${ }^{1,2}$, George Loudos ${ }^{1}$ \\ From PSMR 2015: 4th Conference on PET/MR and SPECT/MR \\ La Biodola, Isola d'Elba, Italy. 17-21 May 2015
}

${ }^{1}$ Technological Educational Institute of Athens, Greece
SiPM arrays are insensitive to magnetic fields and thus good candidates for hybrid PET/MR imaging systems. Moreover, due to their small size and flexibility can be used in dedicated small field of view small animal imaging detectors and especially in head PET/MR studies in mice. Co-doped LFS-3 scintillator crystals have higher light yield and slightly faster response than that of LSO:Ce mainly due to the co-doped activation of emission centers with varying materials such as $\mathrm{Ce}, \mathrm{Gd}, \mathrm{Sc}, \mathrm{Y}, \mathrm{La}, \mathrm{Tb}$, or Ca distributed at the molecular scale through the lutetium silicate crystal host. The purpose of this study is to investigate the behavior of the SensL ArraySL-4 $(4 \times 4$ element array of $3 \times 3 \mathrm{~mm}^{2}$ silicon photomultipliers) optical detector coupled to a $6 \times 6$ LFS-3 scintillator array, with $2 \times 2 \times 5 \mathrm{~mm}^{3}$ crystal size elements, for possible applications in small field of view PET/MR imaging detectors. We have designed a symmetric resistive charge division circuit to read out the signal outputs of $4 \times 4$ pixel SiPM array reducing the 16 pixel outputs of the photodetector to 4 position signals. The 4 position signals were digitized using free running Analog to Digital Converters. The ADCs sampling rate was $50 \mathrm{MHz}$. An FPGA (Spartan 6 LX150T) was used for triggering and digital signal processing of the pulses. Experimental evaluation was carried out with ${ }^{22} \mathrm{Na}$ radioactive source and the parameters studied where energy resolution and peak to valley ratio. The first preliminary results of the evaluation shows a clear visualization of the discrete $2 \times 2 \times 5 \mathrm{~mm}^{3}$ LFS-3 scintillator elements. The mean peak to valley ratio of the horizontal profiles on the raw image was measured equal to 11 while the energy resolution was calculated equal to $30 \%$ at the central pixels.

Authors' details

${ }^{1}$ Technological Educational Institute of Athens, Greece. ${ }^{2}$ Department of Medical School, University of Thessaly, Larissa, Greece.

Published: 18 May 2015

doi:10.1186/2197-7364-2-S1-A9

Cite this article as: David et al:: Evaluation of a SiPM array detector coupled to a LFS-3 pixellated scintillator for PET/MR applications. EJNMMI Physics 2015 2(Suppl 1):A9.

\section{SpringerOpen ${ }^{\odot}$}

(C) 2015 David et al; licensee Springer. This is an Open Access article distributed under the terms of the Creative Commons Attribution License (http://creativecommons.org/licenses/by/4.0), which permits unrestricted use, distribution, and reproduction in any medium, provided the original work is properly cited. 